\title{
DUAL CHARACTERIZATIONS OF RELATIVE CONTINUITY OF CONVEX FUNCTIONS
}

\author{
J. BENOIST and A. DANIILIDIS
}

(Received 6 March 2000; revised 20 September 2000)

Communicated by G. Willis

\begin{abstract}
Various properties of continuity for the class of lower semicontinuous convex functions are considered and dual characterizations are established. In particular, it is shown that the restriction of a lower semicontinuous convex function to its domain (respectively, domain of subdifferentiability) is continuous if and only if its subdifferential is strongly cyclically monotone (respectively, $\sigma$-cyclically monotone).
\end{abstract}

2000 Mathematics subject classification: primary 47H05; secondary 52A41, $26 \mathrm{~A} 15$.

Keywords and phrases: convexity, continuity, subdifferential, cyclic monotonicity.

\section{Introduction}

Let $X$ be a Banach space and $f: X \rightarrow \mathbb{R} \cup\{+\infty\}$ a lower semicontinuous (in short Isc) function. A recent result of Correa, Jofre and Thibault [3] asserts that $f$ is convex if and only if its Clarke-Rockafellar subdifferential $\partial f$ is monotone. The same equivalence has also been established for abstract notions of subdifferentials (see [1], for example). In the aforementioned cases, since any notion of subdifferential of a convex function coincides with the classical Fenchel-Moreau subdifferential, it follows that $\partial f$ is not only monotone, but also cyclically monotone (see [6], for example). This latter property (that is, cyclicity) is not just a stronger property than mere monotonicity, but it expresses a behaviour of certain type. This behaviour has already been discussed in relation with integration problems ([2], for example) as well as in generalized convexity [5].

While cyclic monotonicity describes the behaviour of an operator around a 'cycle' of finite points, a variant of it-called $\sigma$-cyclic monotonicity-was introduced and 
studied in [4]. Compared with cyclic monotonicity, this new property carries additional information on the operator, since it describes its behaviour along infinite cycles formed by converging sequences. In particular, the fact that the subdifferential $\partial f$ of a function $f$ is $\sigma$-cyclically monotone guarantees a certain continuity property for the lsc convex function $f$.

In this article we show that the restriction of a lsc convex function to its domain of subdifferentiability is continuous if and only if its subdifferential is $\sigma$-cyclically monotone; (this result was conjectured in [4]). We also introduce the strong cyclic monotonicity, and show that this property characterizes the subdifferentials of the lsc convex functions having a continuous restriction to their domain, see Section 4 .

The paper is organized as follows. In Section 2 we give some preliminary results and we fix our notation. In Section 3 we prove a local version of Rockafellar's formula [8] concerning the representation of the lsc convex functions. This local representation-apart from its independent interest-will be in use in Section 4, where we establish dual characterizations of the continuity of the functions $\left.f\right|_{\operatorname{dom} f}$ and $\left.f\right|_{\text {dom } \partial f}$ for a lsc convex function $f$. Finally, in the same section, we give some criteria for an operator to be strongly cyclically monotone (respectively, $\sigma$-cyclically monotone) and we classify the various concepts of cyclic monotonicity.

\section{Preliminaries}

In the sequel, $X$ will denote a Banach space and $X^{*}$ its dual. For any $x \in X$ and $x^{*} \in X^{*}$ we denote by $\left\langle x^{*}, x\right\rangle$ the value of $x^{*}$ at $x$. For $x \in X$ and $\varepsilon>0$ we denote by $B(x, \varepsilon)$ the closed ball centered at $x$ with radius $\varepsilon>0$, while for $x, y \in X$ we denote by $[x, y]$ the closed segment $\{t x+(1-t) y: t \in[0,1]\}$. For any closed segment $[x, y]$ in $X$ and any $\varepsilon>0$ we denote by $B([x, y], \varepsilon)$ the $\varepsilon$-neighbourhood of the segment $[x, y]$, that is,

$$
B([x, y], \varepsilon):=\{w \in X: \exists z \in[x, y] \text { with }\|z-w\| \leq \varepsilon\} .
$$

Given a function $f: X \rightarrow \mathbb{R} \cup\{+\infty\}$, we denote by $\operatorname{dom} f:=\{x \in X:$ $f(x) \in \mathbb{R}$ \} its domain. We say that $f$ is continuous (respectively $l s c$ ), if it is continuous (respectively lsc) at every point $x \in X$, where $\mathbb{R} \cup\{+\infty\}$ is equipped with the topology generated by the family $\left.\left.\Im_{\mathbb{R}} \cup\{] x,+\infty\right], x \in \mathbb{R}\right\}\left(\Im_{\mathbb{R}}\right.$ being the usual topology of $\mathbb{R}$ ). Note that such functions may take infinite values, as for instance the function $f: \mathbb{R} \rightarrow \mathbb{B} \cup\{+\infty\}$ with $f(x)=1 / x$ if $x>0$ and $+\infty$ if $x \leq 0$.

Concurrently, a function $f$ is said to have a continuous restriction to a subset $S$ of its domain, if $\left.f\right|_{s}$ is a continuous (real-valued) function, see also [7, page 82].

Throughout this article we shall deal with proper (that is, not identically equal to $\{+\infty\})$ lsc convex functions. Let us remark that the class of lsc convex functions 
with a continuous restriction in their domain is much larger than the one of convex continuous functions. It contains in particular all indicator functions of closed convex sets, as well as many other non-continuous functions, see [6, Example 3.8 (a)].

We recall from [6] that the subdifferential $\partial f$ of the function $f$ at a point $x \in \operatorname{dom} f$ is defined as follows

$$
\partial f(x)=\left\{x^{*} \in X^{*}: f(y)-f(x) \geq\left\langle x^{*}, y-x\right\rangle, \forall y \in X\right\} .
$$

Finally, $T: X \rightrightarrows X^{*}$ will denote a multivalued operator defined on $X$ and taking as values subsets of $X^{*}$. We denote by dom $T:=\{x \in X: T(x) \neq \emptyset\}$ its domain. We recall that $T$ is cyclically monotone if for any $n \in \mathbb{N}$, for any $x_{0}, x_{1}, \ldots, x_{n}$ in $X$ and for any $x_{0}^{*} \in T\left(x_{0}\right), x_{1}^{*} \in T\left(x_{1}\right), \ldots, x_{n}^{*} \in T\left(x_{n}\right)$ we have

$$
\sum_{i=0}^{n}\left\langle x_{i}^{*}, x_{i+1}-x_{i}\right\rangle \leq 0,
$$

where $x_{n+1}:=x_{0}$. Moreover, if $T$ is not strictly contained (in the graph sense) in any other cyclically monotone operator, then it is called maximal cyclically monotone. Typical (and in fact exclusive) examples of maximal cyclically monotone operators are the subdifferentials $\partial f$ of convex lsc functions (see [6], for example).

\section{Representation of convex functions}

Rockafellar has proved in [8] that if $T$ is cyclically monotone, then there exists a lsc convex function $f$ such that $T \subseteq \partial f$. The proof of this result involves a typical construction based on $T$. In particular, starting from any point $x_{0}$ of the domain of $T$ (which is supposed to be nonempty), he defined the following lsc convex function $f_{T}$

$$
f_{T}(x)=\sup \left\{\left\langle x_{n}^{*}, x-x_{n}\right\rangle+\sum_{i=0}^{n-1}\left\langle x_{i}^{*}, x_{i+1}-x_{i}\right\rangle\right\}+c,
$$

where $c$ is an arbitrary constant and the supremum is taken over all $n \geq 1$, all $x_{1}, x_{2}, \ldots, x_{n}$ in $\operatorname{dom} T$ and $x_{0}^{*} \in T\left(x_{0}\right), x_{1}^{*} \in T\left(x_{1}\right), \ldots, x_{n}^{*} \in T\left(x_{n}\right)$.

Let us note that $x_{0}$ appears in all sums at the right hand side of (3) and that cyclic monotonicity property guarantees that $f_{T}\left(x_{0}\right)=c$, hence, in particular, dom $f_{T} \neq \emptyset$.

It is proved in [8] that the function $f_{T}$ is unique up to a constant, whenever the operator $T$ is maximal cyclically monotone, in which case $T=\partial f_{T}$.

Applying this result to the maximal cyclically monotone operator $\partial f,(3)$ yields (for $c=f\left(x_{0}\right)$ ) the following representation for the lsc convex function $f$

$$
f(x)=\sup \left\{\left\langle x_{n}^{*}, x-x_{n}\right\rangle+\sum_{i=0}^{n-1}\left\langle x_{i}^{*}, x_{i+1}-x_{i}\right\rangle\right\}+f\left(x_{0}\right),
$$


where the supremum is taken over all $n \geq 1$, all $x_{1}, x_{2}, \ldots, x_{n}$ in dom $\partial f$ and all $x_{0}^{*} \in \partial f\left(x_{0}\right), x_{1}^{*} \in \partial f\left(x_{1}\right), \ldots, x_{n}^{*} \in \partial f\left(x_{n}\right)$.

This representation is global, in the sense that there is no limitation for the choice of the points $x_{1}, x_{2}, \ldots, x_{n}$ in the above supremum. Let us now produce a localized version of the formula (4) which will be useful in the sequel.

PROPOSITION 1. Let $x_{0} \in \operatorname{dom} \partial f$. For any $x \in X$ and $\varepsilon>0$ we have

$$
f(x)=f\left(x_{0}\right)+\sup \left\{\left\langle x_{n}^{*}, x-x_{n}\right\rangle+\sum_{i=0}^{n-1}\left\langle x_{i}^{*}, x_{i+1}-x_{i}\right\rangle\right\},
$$

where the supremum is taken over all $n \geq 1$, all

$$
x_{1}, x_{2}, \ldots, x_{n} \text { in } \operatorname{dom} \partial f \cap B\left(\left[x_{0}, x\right], \varepsilon\right)
$$

and all $x_{0}^{*} \in \partial f\left(x_{0}\right), x_{1}^{*} \in \partial f\left(x_{1}\right), \ldots, x_{n}^{*} \in \partial f\left(x_{n}\right)$.

Note that comparing with (4), the choice of the points $x_{1}, x_{2}, \ldots, x_{n}$ is constrained into the $\varepsilon$-neighbourhood of $\left[x_{0}, x\right]$.

PrOOF. Let us consider the indicator function $h$ of the closed set $B\left(\left[x_{0}, x\right], \varepsilon\right)$, given by

$$
h(y)= \begin{cases}0 & \text { if } y \in B\left(\left[x_{0}, x\right], \varepsilon\right) \\ +\infty & \text { if } y \notin B\left(\left[x_{0}, x\right], \varepsilon\right) .\end{cases}
$$

Since $B\left(\left[x_{0}, x\right], \varepsilon\right)$ is convex, for every $y \in B\left(\left[x_{0}, x\right], \varepsilon\right)$ and $y^{*} \in \partial h(y)$ we have

$$
\left\langle y^{*}, x^{\prime}-y\right\rangle \leq 0, \forall x^{\prime} \in B\left(\left[x_{0}, x\right], \varepsilon\right) .
$$

Let us now consider the lsc convex function $g(y)=f(y)+h(y)$ and let us remark that $\operatorname{dom} \partial g \subset \operatorname{dom} g \subset B\left(\left[x_{0}, x\right], \varepsilon\right)$. Since $\operatorname{dom} f \cap \operatorname{int} \operatorname{dom} h \neq \emptyset$, it follows [6, Proposition 3.15] that

$$
\partial g(y)=\partial f(y)+\partial h(y)
$$

for all $y \in B\left(\left[x_{0}, x\right], \varepsilon\right)$.

Applying formula (4) for the Isc convex function $g$ at the point $x$, and using the fact that $g\left(y^{\prime}\right)=f\left(y^{\prime}\right)$ for all $y^{\prime} \in B\left(\left[x_{0}, x\right], \varepsilon\right)$, we get

$$
f(x)=f\left(x_{0}\right)+\sup \left\{\left\langle x_{n}^{*}, x-x_{n}\right\rangle+\sum_{i=0}^{n-1}\left\langle x_{i}^{*}, x_{i+1}-x_{i}\right\rangle\right\},
$$


where the supremum is taken over all $n \geq 1$, all $x_{1}, x_{2}, \ldots, x_{n}$ in dom $\partial g$ and all $x_{0}^{*} \in \partial g\left(x_{0}\right), x_{1}^{*} \in \partial g\left(x_{1}\right), \ldots, x_{n}^{*} \in \partial g\left(x_{n}\right)$.

In particular, for any $M<f(x)-f\left(x_{0}\right)$, there exist $x_{1}, x_{2}, \ldots, x_{n}$ in $B\left(\left[x_{0}, x\right], \varepsilon\right)$ and $x_{0}^{*} \in \partial g\left(x_{0}\right), x_{1}^{*} \in \partial g\left(x_{1}\right), \ldots, x_{n}^{*} \in \partial g\left(x_{n}\right)$, such that

$$
M<\left\langle x_{n}^{*}, x-x_{n}\right\rangle+\sum_{i=0}^{n-1}\left\langle x_{i}^{*}, x_{i+1}-x_{i}\right\rangle .
$$

Using (8), for $i=0,1, \ldots, n$, we can write $x_{i}^{*}=z_{i}^{*}+y_{i}^{*}$, where $z_{i}^{*} \in \partial f\left(x_{i}\right)$ and $y_{i}^{*} \in \partial h\left(x_{i}\right)$. It now follows from (7) that

$$
M<\left\langle z_{n}^{*}, x-x_{n}\right\rangle+\sum_{i=0}^{n-1}\left\langle z_{i}^{*}, x_{i+1}-x_{i}\right\rangle .
$$

Since $M$ is arbitrarily chosen, the proof is complete.

\section{Main results}

In this section we establish dual characterizations for the class of proper lsc convex functions $f$ such that $\left.f\right|_{\operatorname{dom} \partial f}$ (respectively $\left.f\right|_{\operatorname{dom} f}$ ) is continuous. These characterizations involve properties stronger than (but reminiscent of) cyclic monotonicity for the subdifferential $\partial f$.

Let us first state the following interesting result.

PROPOSITION 2. Let $x_{0} \in \operatorname{dom} \partial f$. Then the following are equivalent:

(i) $\left.f\right|_{\operatorname{dom} f}$ is continuous at $x_{0}$;

(ii) $\left.f\right|_{\text {dom af }}$ is continuous at $x_{0}$.

PROOF. We obviously have (i) implies (ii). For the inverse implication, suppose that $\left.f\right|_{\operatorname{dom} f}$ is not continuoua at $x_{0}$. There then exists $\varepsilon>0$ such that for all $i \geq 1$, there exists $x_{i} \in \operatorname{dom} f \cap B\left(x_{0}, 1 / i\right)$ with

$$
\left|f\left(x_{i}\right)-f\left(x_{0}\right)\right|>\varepsilon .
$$

Now for each $i \geq 1$, we may find $y_{i} \in \operatorname{dom} \partial f \cap B\left(x_{0}, 2 / i\right)$ with

$$
\left|f\left(x_{i}\right)-f\left(y_{i}\right)\right|<\frac{\varepsilon}{2}
$$

(this is possible because $\operatorname{dom} \partial f$ is graphically dense in $\operatorname{dom} f$ ). Then we have $\left\{y_{i}\right\} \rightarrow x_{0}$ and $\left|f\left(y_{i}\right)-f\left(x_{0}\right)\right|>\varepsilon / 2$ for all $i \geq 1$, which contradicts (ii). 


\subsection{Relative continuity on dom $\partial f$}

We recall from [4] the following definition.

DEFINITION 3. (i) Let $x_{0} \in X$. An operator $T$ is called $\sigma$-cyclically monotone at $x_{0}$, if

$$
\limsup _{n \rightarrow \infty} \sum_{i=0}^{n}\left\langle x_{i}^{*}, x_{i+1}-x_{i}\right\rangle \leq 0
$$

for all $x_{0}^{*} \in T\left(x_{0}\right)$, for all $\left\{x_{i}\right\}_{i \geq 1}$ in dom $T$ satisfying $\lim _{i \rightarrow \infty} x_{i}=x_{0}$ and all $\left\{x_{i}^{*}\right\}_{i \geq 1}$ in $X^{*}$ satisfying $x_{i}^{*} \in T\left(x_{i}\right)$ for all $i \geq 1$.

(ii) The operator $T$ is called $\sigma$-cyclically monotorts, if it is $\sigma$-cyclically monotone at every $x_{0} \in X$.

Let us note that Definition 3 implies (in a trivial way) that $T$ is $\sigma$-cyclically monotone at any $x \notin \operatorname{dom} T$.

It is also easily seen that every $\sigma$-cyclically monotone operator is cyclically monotone. Indeed, given points $x_{0}, x_{1}, \ldots, x_{n}$ in dom $T$, it suffices to consider the sequence $\left\{x_{i}\right\}_{i \geq 1}$ in dom $T$, where $x_{i}=x_{0}$, for $i \geq n+1$. Then relation (10) clearly yields (2).

The following theorem gives a positive answer to a conjecture raised in [4].

THEOREM 4. Let $x_{0} \in \operatorname{dom} \partial f$. The following are equivalent:

(i) $\left.f\right|_{\text {dom af }}$ is continuous at $x_{0}$;

(ii) $\partial f$ is $\sigma$-cyclically monotone at $x_{0}$.

Proof. (i) implies (ii). Let $\left\{x_{i}\right\}_{i \geq 1}$ be any sequence in dom $\partial f$ such that $\lim _{i \rightarrow \infty} x_{i}=$ $x_{0}$. For any $x_{i}^{*} \in \partial f\left(x_{i}\right)$, relation (1) implies

$$
f\left(x_{i+1}\right)-f\left(x_{i}\right) \geq\left\langle x_{i}^{*}, x_{i+1}-x_{i}\right\rangle .
$$

Adding (11) from $i=0$ to an arbitrary integer $n$ we obtain

$$
f\left(x_{n+1}\right)-f\left(x_{0}\right) \geq \sum_{i=0}^{n}\left\langle x_{i}^{*}, x_{i+1}-x_{i}\right\rangle .
$$

As $n \rightarrow+\infty$, the continuity of $\left.f\right|_{\text {dom } \partial f}$ at $x_{0}$ yields (10).

(ii) implies (i). Suppose that $\left.f\right|_{\text {dom af }}$ is not continuous at $x_{0}$. Since $f$ is lsc at $x_{0}$, we deduce the existence of a sequence $\left\{x_{i}\right\}_{i \geq 1}$ in dom $\partial f$, such that $x_{i} \rightarrow x_{0}$ and $\liminf _{i \rightarrow \infty} f\left(x_{i}\right)>f\left(x_{0}\right)$. Take any $\varepsilon>0$ such that

$$
\liminf _{i \rightarrow+\infty} f\left(x_{i}\right) \geq f\left(x_{0}\right)+2 \varepsilon .
$$

Let us fix $i \in \mathbb{N}$. Using Proposition 1 for the points $x_{i} \in \operatorname{dom} \partial f$ and $x_{i+1} \in X$ and for the number $1 /(i+1)>0$, we deduce the existence of a finite sequence 
$y_{i, 1}, y_{i, 2}, \ldots, y_{i, k_{i}}$ in dom $\partial f \cap B\left(\left[x_{i}, x_{i+1}\right], 1 /(i+1)\right)$ and of $y_{i, 0}^{*} \in \partial f\left(y_{i, 0}\right), y_{i, 1}^{*} \in$ $\partial f\left(y_{i, 1}\right), \ldots, y_{i, k_{i}}^{*} \in \partial f\left(y_{i, k_{i}}\right)$ such that

$$
\sum_{j=0}^{k_{i}}\left\langle y_{i, j}^{*}, y_{i, j+1}-y_{i, j}\right\rangle \geq f\left(x_{i+1}\right)-f\left(x_{i}\right)-\frac{\varepsilon}{2^{i+1}},
$$

where by convention $y_{i, 0}:=x_{i}, y_{i, k_{i}+1}:=x_{i+1}$. Summing (12) from $i=0$ to an arbitrary integer $n$, we obtain

$$
\sum_{i=0}^{n} \sum_{j=0}^{k_{i}}\left\langle y_{i, j}^{*}, y_{i, j+1}-y_{i, j}\right\rangle \geq f\left(x_{n+1}\right)-f\left(x_{0}\right)-\sum_{i=0}^{n} \frac{\varepsilon}{2^{i+1}} .
$$

Taking the upper limit as $n \rightarrow \infty$, the last inequality yields

$$
\underset{n \rightarrow+\infty}{\limsup } \sum_{i=0}^{n} \sum_{j=0}^{k_{i}}\left\langle y_{i, j}^{*}, y_{i, j+1}-y_{i, j}\right) \geq \varepsilon .
$$

Since the sequence $\left\{y_{0,0}, \ldots, y_{0, k_{0}}, y_{1,0}, \ldots, y_{1, k_{1}}, \ldots\right\}$ is norm converging to $x_{0}$, we conclude that $\partial f$ is not $\sigma$-cyclically monotone.

We can easily deduce the following corollary.

COROLLARY 5. The following statements are equivalent:

(i) $\left.f\right|_{\text {dom af }}$ is continuous;

(ii) $\partial f$ is $\sigma$-cyclically monotone.

We state below some typical examples of lsc convex functions such that $\left.f\right|_{\text {dom af }}$ is discontinuous.

EXAMPLE 1 ([7, page 83]). Let the function $f: \mathbb{R}^{2} \rightarrow \mathbb{R} \cup\{+\infty\}$ be defined by

$$
f\left(x_{1}, x_{2}\right)= \begin{cases}x_{2}^{2} / x_{1} & \text { if } x_{1}>0 \\ 0 & \text { if } x_{1}=x_{2}=0 \\ +\infty & \text { elsewhere. }\end{cases}
$$

The above function is lsc and convex (the latter can be verified by calculating the Hessian). Considering the sequence $\left(1 / n^{3}, 1 / n\right)$ we conclude that $\left.f\right|_{\operatorname{dom} \partial f}$ is not continuous at $(0,0)$.

EXAMPLE 2. Let the function $f: \ell^{2}(\mathbb{N}) \rightarrow \mathbb{B} \cup\{+\infty\}$ be defined by

$$
f(x)=\|x\|_{1}:=\sum_{i=0}^{+\infty}\left|x_{i}\right|
$$


for every $x=\left\{x_{i}\right\} \in \ell^{2}(\mathbb{N})$. Since $f$ is the pointwise supremum of the convex continuous functions $f_{n}: \ell^{2}(\mathbb{N}) \rightarrow \mathbb{R} \cup\{+\infty\}$ defined for all $x=\left\{x_{i}\right\} \in \ell^{2}(\mathbb{N})$ by $f_{n}(x)=\sum_{i=0}^{n}\left|x_{i}\right|$, it is obviously convex and lsc. However, $\left.f\right|_{\operatorname{dom} \partial f}$ is discontinuous at any point of its domain. (Note that in this example the domain of the function $f$ is dense).

\subsection{Relative continuity on $\operatorname{dom} f$}

Let us first give the following definition.

DEFINITION 6. Let $x_{0} \in X$. An operator $T$ is called strongly cyclically monotone at $x_{0}$, if for every $\varepsilon>0$ there exists $\delta>0$ such that for every $x_{1} \in \operatorname{dom} T \cap B\left(x_{0}, \delta\right)$, for every sequence $\left\{x_{i}\right\}_{i \geq 2}$ in dom $T$ satisfying $\lim _{i \rightarrow \infty} x_{i}=x_{0}$, and for every sequence $\left\{x_{i}^{*}\right\}_{i \geq 1}$ in $X^{*}$ satisfying $x_{i}^{*} \in T\left(x_{i}\right)$ for all $i \geq 1$, we have

$$
\limsup _{n \rightarrow \infty} \sum_{i=1}^{n}\left\langle x_{i}^{*}, x_{i+1}-x_{i}\right\rangle \leq \varepsilon .
$$

Let us note that $T$ is strongly cyclically monotone (in a trivial way) at every point in the complement of $\overline{\operatorname{dom} T}$.

PROPOSITION 7. Let $x_{0} \in X$. If $T$ is strongly cyclically monotone at $x_{0}$, then $T$ is also $\sigma$-cyclically monotone at $x_{0}$. The converse is true whenever $x_{0} \in \operatorname{dom} T$.

PROOF. Suppose that $T$ is strongly cyclically monotone at $x_{0}$. It suffices to consider only the case $x_{0} \in \operatorname{dom} T$. If $\left\{x_{i}\right\}_{i \geq 1}$ is any sequence in dom $T$ such that $\lim _{i \rightarrow \infty} x_{i}=$ $x_{0}$, then for any $\varepsilon>0$ we can apply (13) for the sequence $\left\{y_{i}\right\}_{i \geq 1}$ defined by $y_{1}:=x_{0}$ and $y_{i}:=x_{i-1}$ for all $i \geq 2$ (note that $y_{1} \in \operatorname{dom} T \cap B\left(x_{0}, \delta\right)$ for all $\delta$ ). Since $\varepsilon$ is arbitrary, we easily conclude that (10) is verified, hence $T$ is $\sigma$-cyclically monotone.

Conversely, suppose that $x_{0} \in \operatorname{dom} T$ and that $T$ is $\sigma$-cyclically monotone at $x_{0}$. We shall show that $T$ is strongly cyclically monotone at $x_{0}$. Let $\varepsilon>0$. Then pick any $x_{0}^{*}$ in $T\left(x_{0}\right)$ and set $\delta=\varepsilon /\left\|x_{0}^{*}\right\|$ (if $x_{0}^{*}=0$, then take $\delta=1$ ). Then for every $x_{1} \in \operatorname{dom} T \cap B\left(x_{0}, \delta\right)$, we have

$$
\left|\left\langle x_{0}^{*}, x_{1}-x_{0}\right\rangle\right| \leq \varepsilon .
$$

Since $T$ is $\sigma$-cyclically monotone at $x_{0}$, it follows that for every sequence $\left\{x_{i}\right\}_{i \geq 2}$ in dom $T$ satisfying $\lim _{i \rightarrow \infty} x_{i}=x_{0}$, and for every sequence $\left\{x_{i}^{*}\right\}_{i \geq 1}$ in $X^{*}$ satisfying $x_{i}^{*} \in T\left(x_{i}\right)$ for all $i \geq 1$, we have

$$
\limsup _{n \rightarrow \infty} \sum_{i=0}^{n}\left\langle x_{i}^{*}, x_{i+1}-x_{i}\right\rangle \leq 0 .
$$

Combining (14) and (15) we conclude that $T$ is strongly cyclically monotone at $x_{0}$. 
Consider now the case where $T=\partial f$. Since $\overline{\operatorname{dom} f}=\overline{\operatorname{dom} \partial f}$, we conclude that $\partial f$ is $\sigma$-cyclically monotone at every $x_{0} \in X \backslash$ dom $\partial f$ and strongly cyclically monotone at every $x_{0} \in X \backslash \overline{\operatorname{dom} f}$. It now follows from Proposition 7 that for any $x_{0} \in \operatorname{dom} \partial f, \partial f$ is strongly cyclically monotone at $x_{0}$ if and only if $\partial f$ is $\sigma$-cyclically monotone at $x_{0}$ (if and only if $\left.f\right|_{\text {dom } \partial f}$ is continuous at $x_{0}$ ). The following theorem (analogue to Theorem 4) deals with the case $x_{0} \in \operatorname{dom} f$.

THEOREM 8. Let $x_{0} \in \operatorname{dom} f$. The following are equivalent:

(i) $\left.f\right|_{\operatorname{dom} f}$ is continuous at $x_{0}$;

(ii) $\partial f$ is strongly cyclically monotone at $x_{0}$.

PROOF. (i) implies (ii). Let $\varepsilon>0$. According to (i), there exists $\delta>0$ such that for all $x \in \operatorname{dom} \partial f \cap B\left(x_{0}, \delta\right)$

$$
\left|f(x)-f\left(x_{0}\right)\right|<\varepsilon .
$$

Fix any $x_{1}$ in dom $\partial f \cap B\left(x_{0}, \delta\right)$. Consider now any sequence $\left\{x_{i}\right\}_{i \geq 1}$ in dom $\partial f$ satisfying $\lim _{i \rightarrow \infty} x_{i}=x_{0}$. Then for all $i \geq 1$ and all $x_{i}^{*} \in \partial f\left(x_{i}\right)$, we have

$$
f\left(x_{i+1}\right)-f\left(x_{i}\right) \geq\left\langle x_{i}^{*}, x_{i+1}-x_{i}\right\rangle .
$$

Adding (17) from $i=1$ to an arbitrary integer $n$, we obtain

$$
f\left(x_{n+1}\right)-f\left(x_{1}\right) \geq \sum_{i=1}^{n}\left\langle x_{i}^{*}, x_{i+1}-x_{i}\right\rangle .
$$

As $n \rightarrow+\infty$, the continuity of $\left.f\right|_{\operatorname{dom} f}$ together with (16) yields (13).

(ii) implies (i). Suppose that $\left.f\right|_{\operatorname{dom} f}$ is not continuous at $x_{0}$. Then since $f$ is lsc, there exists $\varepsilon>0$ and $\left\{x_{i}\right\}_{i \geq 1}$ in $\operatorname{dom} f$ satisfying $\lim _{i \rightarrow \infty} x_{i}=x_{0}$ such that

$$
\liminf _{i \rightarrow \infty} f\left(x_{i}\right)>f\left(x_{0}\right)+3 \varepsilon
$$

Since $\operatorname{dom} \partial f$ is graphically dense in $\operatorname{dom} f$ ([6, Theorem 3.17]), without loss of generality we assume that $\left\{x_{i}\right\}_{i \geq 1}$ is in dom $\partial f$. Moreover, for any $\delta>0$, we may choose $x_{1, \delta}$ in dom $\partial f \cap B\left(x_{0}, \delta\right)$ such that

$$
\left|f\left(x_{1, \delta}\right)-f\left(x_{0}\right)\right|<\varepsilon .
$$

It follows that

$$
\liminf _{i \rightarrow \infty} f\left(x_{i}\right)>f\left(x_{1, \delta}\right)+2 \varepsilon .
$$

Applying Proposition 1 successively for the points $\left\{x_{1,8}, x_{1}\right\}$ and $\left\{x_{i}, x_{i+1}\right\}$ for $i \geq 1$, and repeating the arguments of the final part of the proof of Theorem 4 ((ii) implies (i)) 
we deduce the existence of a sequence $\left\{z_{i}\right\}_{i}$ in $X$ satisfying $z_{0}:=x_{1, \delta}$ and $\lim _{i \rightarrow \infty} z_{i}=$ $x_{0}$, and of a sequence $\left\{z_{i}^{*}\right\}_{i \geq 1}$ satisfying $z_{i}^{*} \in \partial f\left(z_{i}\right)$ for all $i$, such that for every $n$ we have

$$
\sum_{i=0}^{\varphi(n)}\left\langle z_{i}^{*}, z_{i+1}-z_{i}\right\rangle \geq f\left(x_{n+1}\right)-f\left(x_{1, \delta}\right)-\varepsilon,
$$

for some increasing function $\varphi: \mathbb{N} \rightarrow \mathbb{N}$. As $n \rightarrow \infty$, thanks to (19), we obtain

$$
\limsup _{n \rightarrow \infty} \sum_{i=0}^{n}\left\langle z_{i}^{*}, z_{i+1}-z_{i}\right\rangle>\varepsilon .
$$

Thus $\partial f$ is not strongly cyclically monotone at $x_{0}$.

We shall say that $\partial f$ is strongly cyclically monotone on $\operatorname{dom} f$ if it is strongly cyclically monotone at every point of $\operatorname{dom} f$. The following corollary is analogue to Corollary 5.

COROLLARY 9. The following are equivalent:

(i) $\left.f\right|_{\operatorname{dom} f}$ is continuous;

(ii) $\partial f$ is strongly cyclically monotone on $\operatorname{dom} f$.

REMARK. Combining Proposition 2 with Theorem 4 and Theorem 8 we obtain an indirect way to establish Proposition 7 for the special case $T=\partial f$.

Finally, the following proposition shows that $\partial f$ is not strongly monotone at any $x_{0} \in \overline{\operatorname{dom} f} \backslash \operatorname{dom} f$.

Proposition 10. Let $x_{0} \in \overline{\operatorname{dom} f} \backslash \operatorname{dom} f$. Then $\partial f$ is not strongly cyclically monotone at $x_{0}$.

PROOF. Since $f$ is lsc and $x_{0} \notin \operatorname{dom} f$, it follows that for any sequence $\left\{x_{i}\right\}_{i \geq 1}$ in dom $\partial f$ satisfying $\lim _{i \rightarrow \infty} x_{i}=x_{0}$ we have $\lim _{i \rightarrow \infty} f\left(x_{i}\right)=+\infty$. Using the same arguments as in the proof of Theorem $8((i i)$ implies $(i))$, for every $M>0$ we obtain the existence of a sequence $\left\{z_{i}\right\}_{i \geq 1}$ in $X$ satisfying $z_{1}:=x_{1}$ and $\lim _{i \rightarrow \infty} z_{i}=x_{0}$, and of a sequence $\left\{z_{i}^{*}\right\}_{i \geq 1}$ satisfying $z_{i}^{*} \in \partial f\left(z_{i}\right)$ for all $i \geq 1$, such that for $n$ large enough

$$
\sum_{i=1}^{n}\left\langle z_{i}^{*}, z_{i+1}-z_{i}\right\rangle \geq M .
$$

Thus $\partial f$ cannot be strongly cyclically monotone at $x_{0}$.

Let us now show that Theorem 4 and Theorem 8 characterize different classes of functions. This is illustrated in the following example. 
EXAMPLE. Consider the following proper lsc convex function $f: \mathbb{R}^{2} \rightarrow \mathbb{R} \cup\{+\infty\}$ defined by

$$
f\left(x_{1}, x_{2}\right)= \begin{cases}x_{2}^{2} / x_{1}-\sqrt{x_{1}} & \text { if } x_{1}>0 \\ 0 & \text { if } x_{1}=x_{2}=0 \\ +\infty & \text { elsewhere }\end{cases}
$$

It is easily seen that

$$
\operatorname{dom} \partial f=\left\{\left(x_{1}, x_{2}\right) \in \mathbb{R}^{2}: x_{1}>0\right\}
$$

while

$$
\operatorname{dom} f=\operatorname{dom} \partial f \cup\{(0,0)\} .
$$

Clearly, the function $\left.f\right|_{\operatorname{dom} \partial f}$ is continuous. On the other hand, the function $\left.f\right|_{\operatorname{dom} f}$ is discontinuous at $(0,0)$, as can be shown by considering the sequence $\left(1 / n^{3}, 1 / n\right)$ which converges to $(0,0)$.

REMARK. The above example exhibits in particular the difference between Definition 3 and Definition 6 for $x_{0} \in \overline{\operatorname{dom} T} \backslash \operatorname{dom} T$ (compare also with Proposition 2).

\subsection{Classification of the various concepts of cyclic monotonicity}

We first give the following definitions.

DEFINITION 11 . We say that

(i) $\partial f$ is locally bounded on $\operatorname{dom} f$, if for every $x_{0} \in \operatorname{dom} f$ there exist $M>0$ and $\delta>0$ such that

$$
\forall z \in \operatorname{dom} \partial f \cap B\left(x_{0}, \delta\right), \forall z^{*} \in \partial f(z),\left\|z^{*}\right\| \leq M .
$$

(ii) $\partial f$ has a locally bounded selection on $\operatorname{dom} f$, if for every $x_{0} \in \operatorname{dom} f$ there exist $M>0$ and $\delta>0$ such that

$$
\forall z \in \operatorname{dom} \partial f \cap B\left(x_{0}, \delta\right), \quad \exists z^{*} \in \partial f(z), \quad\left\|z^{*}\right\| \leq M .
$$

Let us observe that if $f$ is the indicator function of any closed convex subset $K$ of $X$, then the operator $\partial f$ has a locally bounded selection on $\operatorname{dom} f$, without being locally bounded (unless $K=X$ ).

The following result is well known (see [6], for example).

THEOREM 12. $f$ is continuous if and only if $\partial f$ is locally bounded on $\operatorname{dom} f$. 
In the above case, it follows that $\operatorname{dom} f$ is open and $\operatorname{dom} f=\operatorname{dom} \partial f$. Let us remark that it is possible to have $\operatorname{dom} f \neq X$. It suffices to consider the function $f: \mathbb{R} \rightarrow \mathbb{R} \cup\{+\infty\}$ with

$$
f(x)= \begin{cases}+\infty & \text { if } x \leq 0 \\ 1 / x & \text { if } x>0\end{cases}
$$

We now state the following sufficient condition for strong cyclic monotonicity.

PROPOSITION 13. If $\partial f$ has a locally bounded selection on $\operatorname{dom} f$, then $\partial f$ is strongly cyclically monotone on $\operatorname{dom} f$.

ProOF. Let $x_{0} \in \operatorname{dom} f$. In view of Theorem 8 it suffices to show that the function $\left.f\right|_{\text {dom } f}$ is continuous at $x_{0}$. Since $\partial f$ has a locally bounded selection on $\operatorname{dom} f$ and since $\operatorname{dom} \partial f$ is dense in $\operatorname{dom} f$, there exists $\delta>0$ such that for every $x \in$ $\operatorname{dom} f \cap B\left(x_{0}, \delta\right)$ we can find a sequence $\left\{x_{i}\right\}_{i \geq 1}$ in dom $\partial f$ satisfying $\lim _{i \rightarrow \infty} x_{i}=x$ and a sequence $\left\{x_{i}^{*}\right\}_{i \geq 1}$ in $X^{*}$ satisfying $x_{i}^{*} \in \partial f\left(x_{i}\right)$ and $\left\|x_{i}^{*}\right\| \leq M$ for all $i \geq 1$. Then (1) yields that

$$
f\left(x_{i}\right) \leq f\left(x_{0}\right)+\left\langle x_{i}^{*}, x_{i}-x_{0}\right\rangle
$$

Since $f$ is Isc, we conclude as $i \rightarrow+\infty$ that

$$
f(x) \leq f\left(x_{0}\right)+M\left\|x_{0}-x\right\| .
$$

Since (22) holds for all $x \in \operatorname{dom} f \cap B\left(x_{0}, \delta\right)$, it follows that

$$
\left.\limsup _{x \rightarrow x_{0}} f\right|_{\operatorname{dom} f}(x) \leq f\left(x_{0}\right) .
$$

Since $f$ is lsc we conclude that $\left.f\right|_{\operatorname{dom} f}$ is continuous at $x_{0}$.

The converse of Proposition 13 is not true as it is shown by the following example.

EXAMPLE. Consider the function $f: \mathbb{R} \rightarrow \mathbb{R} \cup\{+\infty\}$ with

$$
f(x)= \begin{cases}-\sqrt{-x} & \text { if } x \leq 0 \\ +\infty & \text { if } x>0\end{cases}
$$

Then the restriction $\left.f\right|_{\operatorname{dom} f}$ is obviously continuous. On the other hand, $\partial f$ does not have a locally bounded selection (take $x_{0}=0$ ). 
Thus, between the various concepts we have considered, the following implications hold, and none other:

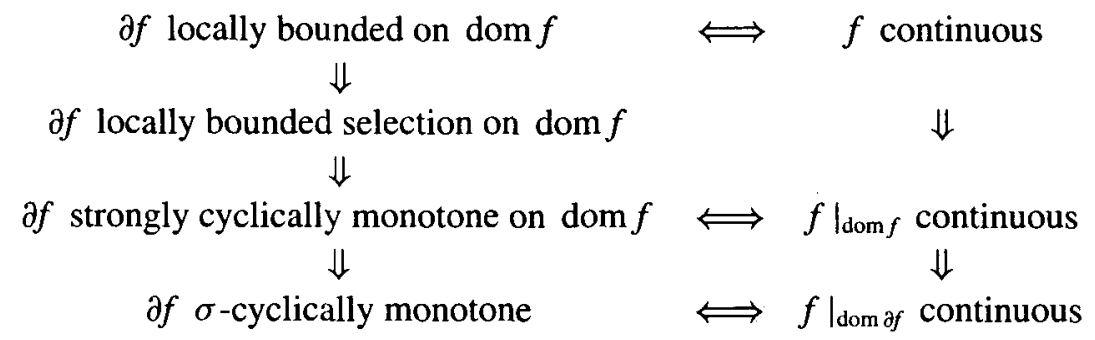

\section{Acknowledgment}

The research of the second author was supported by the TMR post-doctoral grant ERBFMBI CT 983381.

\section{References}

[1] D. Aussel, J.-N. Corvellec and M. Lassonde, 'Mean value property and subdifferential criteria for lower semicontinuous functions', Trans. Amer. Math. Soc. 347 (1995), 4147-4161.

[2] J. Borwein, W. Moors and Y. Shao, 'Subgradient representation of multifunctions', J. Austral. Math. Soc. (Series B) 40 (1998), 1-13.

[3] R. Correa, A. Jofre and L. Thibault, 'Characterization of lower semicontinuous convex functions', Proc. Amer. Math. Soc. 116 (1992), 67-72.

[4] A. Daniilidis, 'Subdifferentials of convex functions and sigma-cyclic monotonicity', Bull. Austral. Math. Soc. 61 (2000), 269-276.

[5] A. Daniilidis and N. Hadjisavvas, 'On the subdifferentials of quasiconvex and pseudoconvex functions and cyclic monotonicity', J. Math. Anal. Appl. 237 (1999), 30-42.

[6] R. Phelps, Convex functions, monotone operators and differentiability, 2nd edition (Springer, Berlin, 1991).

[7] R. T. Rockafellar, Convex analysis (Princeton University Press, Princeton NJ, 1970).

[8] _ ' 'On the maximal monotonicity of subdifferential mappings', Pacific J. Math. 33 (1970), 209-216.

LACO, CNRS UPRES 6090

Faculté des Sciences

Université de Limoges

123, avenue Albert Thomas

87060 Limoges, Cedex

France

e-mail: joel.benoist@unilim.fr
CNRS ERS 2055

Laboratoire de Mathématiques Appliquées Université de Pau et des Pays de l'Adour avenue de l'Université $64000 \mathrm{Pau}$ France e-mail: aris.daniilidis@univ-pau.fr 
